\title{
An essential role for calcium flux in phagocytes for apoptotic cell engulfment and the anti-inflammatory response
}

\author{
MA Gronski ${ }^{1}$, JM Kinchen ${ }^{1}$, IJ Juncadella ${ }^{1}$, NC Franc ${ }^{2}$ and KS Ravichandran ${ }^{\star, 1}$
}

Cells undergo programmed cell death/apoptosis throughout the lifespan of an organism. The subsequent immunologically silent removal of apoptotic cells plays a role in the maintenance of tolerance; defects in corpse clearance have been associated with autoimmune disease. A number of receptors and signaling molecules involved in this process have been identified, but intracellular signaling downstream of corpse recognition is only now being defined. Calcium plays a key role as a second messenger in many cell types, leading to the activation of downstream molecules and eventual transcription of effector genes; however, the role of calcium signaling during apoptotic cell removal is unclear. Here, using studies in cell lines and in the context of a whole organism, we show that apoptotic cell recognition induces both an acute and sustained calcium flux within phagocytes and that the genes required for calcium flux are essential for engulfment. Furthermore, we provide evidence that both the release of calcium from the endoplasmic reticulum and the entry of extracellular calcium through CRAC channels into the phagocytes are important during engulfment. Moreover, knockdown in Caenorhabditis elegans of stim-1 and jph-1, two genes linked to the entry of extracellular calcium into cells, led to increased persistence of apoptotic cells in the nematode. Loss of these genes seemed to affect early signaling events, leading to a decreased enrichment of actin adjacent to the apoptotic cell during corpse removal. We also show that calcium is crucial for the secretion of TGF- $\beta$ by the phagocytes during the engulfment of apoptotic cells. Taken together, these data point to an earlier unappreciated and evolutionarily conserved role for calcium flux at two distinguishable steps: the formation of the phagocytic cup and the internalization of the apoptotic cell, and the anti-inflammatory signaling induced in phagocytes by contact with apoptotic cells.

Cell Death and Differentiation (2009) 16, 1323-1331; doi:10.1038/cdd.2009.55; published online 22 May 2009

In multicellular organisms, engulfment of apoptotic cells is important for the maintenance of tissue homeostasis and the prevention of autoimmunity. ${ }^{1}$ Although classically this process was believed to be performed largely by professional phagocytes, such as macrophages and dendritic cells, more recent data suggest that neighboring cells often remove apoptotic cell corpses. ${ }^{2}$ Recognition of apoptotic cells occurs through receptors on the engulfing cells that recognize the so-called 'eat-me' signals on the surface of the apoptotic cell. $^{3}$ Some of the molecules involved downstream of these receptors have been identified and shown to lead to the activation of the small GTPase Rac1, resulting in the reorganization of the actin cytoskeleton and apoptotic cell engulfment. ${ }^{4}$ Contrary to other types of phagocytosis, recognition of apoptotic cells by phagocytes elicits the production of anti-inflammatory mediators, such as transforming growth factor- $\beta$ (TGF- $\beta$ ) and prostaglandin $\mathrm{E}_{2},{ }^{1}$ which are thought to be a part of the 'immunologically silent' clearance of apoptotic cells and tolerance to self-antigens derived from apoptotic cells. ${ }^{3}$ Interestingly, the 'recognition' of apoptotic cells by phagocytes without physical internalization seems to be sufficient to induce an anti-inflammatory response from macrophages. ${ }^{5}$ This suggests that signals for the internalization of the corpse by phagocytes and for the generation of anti-inflammatory mediators may be separate and diverge downstream of receptor activation. Identifying common versus unique signals for these engulfment events could also help define features relevant for immunological tolerance and autoimmunity.

$\mathrm{Ca}^{2+}$ ions also serve as important second messengers in signal transduction in many cell types, including neurons, lymphocytes, muscle cells, and leukocytes. In many cells of the immune system, triggering of cell surface receptors also induces a rise in cytosolic calcium, which induces the activation of a number of signaling pathways that promote activation, proliferation, and various effector functions in the cell (reviewed in Ref. ${ }^{6}$ ). Cytoplasmic levels of $\mathrm{Ca}^{2+}$ can increase either from release from internal calcium stores

\footnotetext{
${ }^{1}$ Beirne Carter Center for Immunology, Center for Cell Clearance, and the Department of Microbiology, University of Virginia, Charlottesville, VA 22908, USA and ${ }^{2}$ Department of Genetics, The Scripps Research Institute, La Jolla, CA 92037, USA.

*Corresponding author: KS Ravichandran, Carter Immunology Center, University of Virginia, MR4, Rm4072D, Box 801386, 409 Lane Road, Charlottesville, VA 22908, USA. Tel: + 434243 6093; Fax: + 434243 6093; E-mail: ravi@ virginia.edu

Keywords: calcium; engulfment; phagocyte; inflammation

Abbreviations: TGF- $\beta$, transforming growth factor- $\beta$; CRAC channels, calcium release-activated calcium channels; STIM, stromal interaction molecule; $\mathrm{Ca}^{2+}$, calcium ion; TNF- $\alpha$, tumor necrosis factor- $\alpha$; LPS, lipopolysaccharide; ER, endoplasmic reticulum; BAPTA, 1,2-bis(o-aminophenoxy)ethane- $N, N, N, N$-tetraacetic acid; EGTA, ethylyne glycol tetraacetic acid; $\mathrm{Mg}^{2+}$, magnesium ion; SERCA, sarco/endoplasmic reticulum $\mathrm{Ca}^{2+}$-ATPase; TAMRA, carboxytetramethylrhodamine; DMSO, dimethyl sulfoxide; ORAI, ORAI calcium release-activated calcium modulator; siRNA, small interfering RNA; JPH, junctophilin

Received 10.12.08; revised 14.4.09; accepted 15.4.09; Edited by S Nagata; published online 22.5.09
} 
(such as the endoplasmic reticulum (ER)) or entry from outside the cell through calcium channels, leading to the rapid activation of molecules that promote activation, proliferation, and other functions in a cell. Phagocytes also use calcium flux: activation of macrophages with LPS, for example, leads to an increase in cytosolic calcium and the eventual production of TNF $-\alpha{ }^{7}$ However, the role of calcium flux in mammalian phagocytes during the removal of apoptotic cells is not known. During the course of our work, Cuttell et al. ${ }^{8}$ reported that mutations in several genes linked to calcium flux can lead to defects in the proper clearance of apoptotic cells. It was unclear, however, whether recognition of apoptotic cells leads to calcium flux in phagocytes, at what step(s) the phagocyte calcium flux was required in the engulfment process, and whether the requirement for genes linked to calcium flux machinery was evolutionarily conserved. Here, we show that a rise in cytoplasmic $\mathrm{Ca}^{2+}$ occurs during the recognition of apoptotic cells and that it is relevant for efficient engulfment of apoptotic cells by the mammalian cells. Our studies show an evolutionarily conserved role for calcium flux during apoptotic cell clearance in Caenorhabditis elegans and that calcium flux in phagocytes regulates at least two distinct steps during apoptotic cell clearance.

\section{Results}

Intracellular calcium flux in phagocytes after contact with apoptotic cells is essential for the uptake of targets. In many cells of the immune system, triggering of cell surface receptors results in a rise in cytosolic calcium, which activates a number of signaling pathways linked to optimal activation, proliferation, and downstream gene expression. ${ }^{6}$ To determine whether a calcium flux is initiated in phagocytes during the recognition of apoptotic cells, we labeled LR73 cells (a phagocytic fibroblast cell line) with the calcium-binding fluorescent dye Fluo-4, incubated them with apoptotic Jurkat cells, and followed the changes in intracellular calcium levels by real-time fluorimetry. We were able to detect a steady rise in cytosolic $\mathrm{Ca}^{2+}$ after the addition of apoptotic cells (Figure 1a), confirming that calcium flux occurs after the stimulation of phagocytes with apoptotic cells.

a
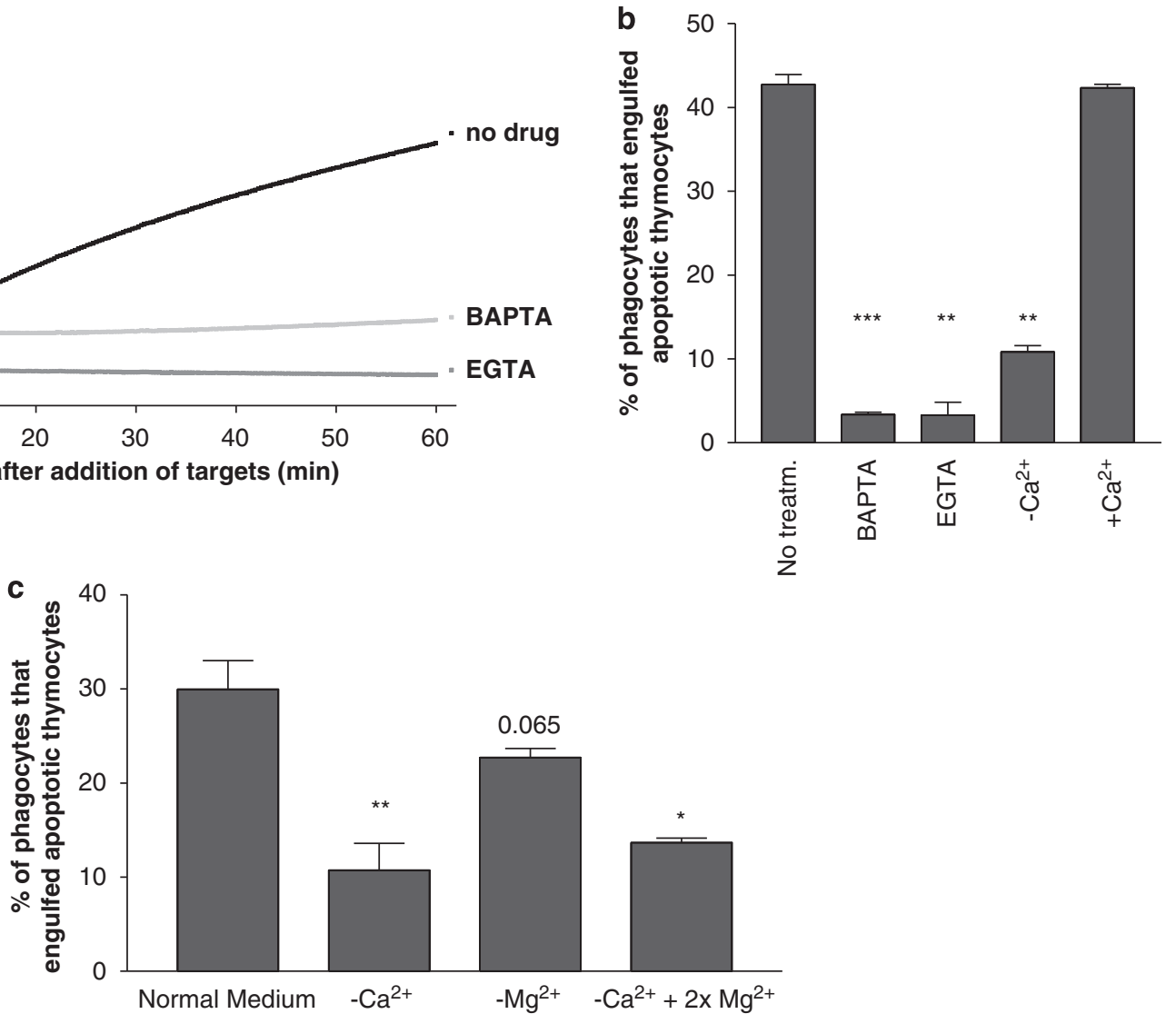

Figure 1 Functional requirement of calcium flux during engulfment of apoptotic cells. (a) Apoptotic Jurkat cells were added (time 0 ) to Fluo-4 labeled LR73 phagocytic cells, and cytoplasmic calcium levels were assessed over time using a fluorescent microplate reader. BAPTA and EGTA were added to LR73 cells 5 min before addition of apoptotic cells. (b) Labeled apoptotic thymocytes were incubated with LR73 cells in the presence or absence of BAPTA or EGTA, as well as in medium with or without calcium, and engulfment was assessed by a flow cytometry-based phagocytosis assay. (c) Labeled apoptotic thymocytes were incubated with LR73 cells in complete fluorimetry medium, medium without $\mathrm{Ca}^{2+}$, medium without $\mathrm{Mg}^{2+}$, or medium without $\mathrm{Ca}^{2+}$ but with two-fold higher $\mathrm{Mg}^{2+}$ concentration so that the total divalent cation concentration in the medium was as in complete medium, and engulfment was assessed by flow cytometry. Significance is indicated as follows: ${ }^{*} P=0.01-0.05 ;{ }^{* *} P=0.001-0.01$; ${ }^{* * *} P<0.001$. All of these experiments were repeated a minimum of three times 
Rise in cytoplasmic levels of $\mathrm{Ca}^{2+}$ in other contexts, such as after antigen receptor stimulation in lymphocytes, occurs as a result of release from internal calcium stores in the ER, followed by the entry of extracellular calcium through calcium channels in the plasma membrane. ${ }^{9}$ Entry of extracellular calcium, which is usually triggered by the sensing of the calcium release from intracellular stores, serves to replenish the depleted stores from the ER and can also critically modulate signal transduction in other systems. ${ }^{10}$ To test whether the observed rise in calcium in phagocytes after incubation with apoptotic cells was because of the entry of calcium from outside, we added BAPTA or EGTA to chelate extracellular calcium present in the cell culture medium. Both drugs inhibited the calcium flux in phagocytes exposed to apoptotic cells (Figure 1a), suggesting a role for extracellular calcium during engulfment.

We next asked whether there was a functional requirement for this calcium flux within the phagocyte during corpse removal. Compared with control conditions in which a large fraction of LR73 cells engulf the targets ( $>40 \%$ ), blocking calcium influx with either EGTA or BAPTA greatly blocked the engulfment (Figure 1b). We did not see obvious changes in the viability or morphology of the phagocytes because of the addition of either BAPTA or EGTA (data not shown). It was possible, however, that these two drugs had unintended secondary effects; to control for this, we performed the engulfment assay with or without calcium in the medium (Figure 1b). In a calcium-free medium, engulfment of apoptotic thymocytes was similarly decreased; importantly, engulfment was restored when $1 \mathrm{mM}$ calcium was added back to the same medium (Figure 1b). Similar results were found with $\mathrm{NIH} / 3 \mathrm{~T} 3$ cells as phagocytes and apoptotic Jurkat cells as targets (data not shown).

Divalent cations are also important for integrin activation; ${ }^{11}$ binding domains on integrins undergo conformational changes on cation binding. ${ }^{12}$ Thus, we tested whether the inhibition of engulfment was an indirect effect of a loss of cations by assessing engulfment in media without $\mathrm{Ca}^{2+}$, without $\mathrm{Mg}^{2+}$, or a medium without $\mathrm{Ca}^{2+}$ but with a two-fold higher concentration of $\mathrm{Mg}^{2+}$ added to keep the number of cations the same. Inhibition of engulfment in the absence of calcium could not be rescued by the addition of extra $\mathrm{Mg}^{2+}$ (Figure 1c). Although the $\mathrm{Mg}^{2+}$-deficient medium had a slight effect on engulfment, it did not significantly decrease apoptotic cell uptake $(P=0.065)$. As a second way of addressing the potential decreased binding by phagocytes to apoptotic thymocytes, we carried out the experiment at $4{ }^{\circ} \mathrm{C}$. Earlier studies have suggested that any TAMRA-positive staining at $4{ }^{\circ} \mathrm{C}$ is the result of binding to but not internalization by phagocytes $^{13}$ and unpublished observations). We found no difference in the degree of binding of apoptotic targets to phagocytes when incubated without drug treatment, with EGTA, or thapsigargin (a sarco/endoplasmic reticulum $\mathrm{Ca}^{2+}$. ATPase (SERCA) inhibitor) at $4^{\circ} \mathrm{C}$ (Supplementary Figure 2). Thus, calcium flux within phagocytes seems to be important for the efficient removal of apoptotic cells, and this effect is not simply because of the effect of a lower concentration of divalent cations on integrin activation.

Two known signaling pathways downstream of the engulfment receptors converge at the small GTPase Rac1 leading to actin cytoskeleton reorganization and apoptotic cell engulfment. ${ }^{4}$ Phagocytic cup formation occurs subsequent to corpse recognition by the phagocyte, which results in Rac activation and the rearrangement of the actin cytoskeleton promoting membrane movement to engulf the target. To characterize the effects of calcium on cell engulfment, we addressed the actin-dependent phagocytic cup formation around apoptotic cells. Chelating calcium with BAPTA resulted in an almost complete inhibition of phagocytic cup formation, similar to the effects seen in the presence of the actin polymerization inhibitor cytochalasin D, whereas the vehicle control, DMSO, had no obvious effect on phagocytic cup formation (Figure $2 \mathrm{a}$ and $\mathrm{b}$ ). Thus, calcium flux within phagocytes seems to be essential for actin polymerization and phagocytic cup formation.

\section{Characterization of the calcium machinery involved} during engulfment. An increase in intracellular calcium levels can be a consequence of release from ER stores, influx of extracellular calcium, and/or release from other organelles (e.g. mitochondria). We used a variety of compounds that have been used earlier to target molecules and/or channels involved in the modulation of calcium flux to test their involvement during the engulfment of apoptotic cells. Thapsigargin is a tight binding inhibitor of SERCA; by blocking the ability of cells to pump calcium into the ER, thapsigargin causes these stores to be depleted. We found that thapsigargin could significantly inhibit engulfment of apoptotic thymocytes by LR73 cells (Figure 3a), suggesting that intracellular calcium stores are important for engulfment. Ru360 binds the mitochondrial calcium uniporter in the mitochondria (a relatively minor source of intracellular calcium) with high affinity and blocks calcium uptake. ${ }^{14}$ Under the conditions of our phagocytosis assay, Ru360 had no detectable effect on engulfment (Figure 3a), suggesting that mitochondria are not a significant source of calcium during the engulfment of apoptotic cells.

The second stage of calcium mobilization in other systems frequently involves a sustained influx of extracellular calcium across the plasma membrane through calcium channels. As seen above in the EGTA and BAPTA experiments (Figure 1b), the entry of extracellular calcium into the phagocytes seems essential during the engulfment of apoptotic cells. To address the role of calcium channels, we used nickel, which at high concentrations blocks all voltage-activated calcium channels. $^{15,16}$ The addition of nickel decreased engulfment, supporting the importance of plasma membrane calcium channels in corpse removal with slightly more inhibition at higher $(100 \mathrm{mM})$ than lower $(1 \mathrm{mM})$ concentrations (Figure 3a). As nickel is a Lewis acid and can change the $\mathrm{pH}$ of the medium, it was possible that low $\mathrm{pH}$ might inhibit engulfment; however, decreasing the $\mathrm{pH}$ of the medium had little effect on engulfment. Thus, calcium channels that permit entry of extracellular calcium into the cell seem important for engulfment.

Various channels on the surface of the cell allow calcium entry into the cytoplasm. $\mathrm{Ca}^{2+}$ release-activated $\mathrm{Ca}^{2+}$ (CRAC) channels are the main store-operated channels and the principal pathway for calcium influx in developing 
and mature T cells. ${ }^{6}$ We used YM-58483 (BTP2), which selectively inhibits CRAC channels. ${ }^{17,18}$ Although lower concentrations of YM-58483 $(10 \mu \mathrm{M})$ had only a small effect on engulfment, higher concentrations $(100 \mu \mathrm{M})$ greatly decreased engulfment. Calcium flux during engulfment in the presence of thapsigargin, YM58483, nickel, or BAPTA is shown in Supplementary Figure 1.

To validate the above drug studies, we used the siRNAmediated knockdown of the ORAI proteins ORAI1, ORAI2, and/or ORAI3, which oligomerize to form CRAC channels on the plasma membrane. ${ }^{19}$ We established that the knockdown targeting individual orai transcripts was specific and that the levels of other orai members were not greatly upregulated (to compensate for knockdown of a given orai member) (Figure 3b). Knockdown of any single ORAl homolog decreased engulfment, with a greater effect for the knockdown of ORAI1 and ORAI2 (Figure 3c). Knockdown of all three ORAI homologs reduced engulfment even further (Figure 3c). Thus, CRAC channels seem to be an important pathway through which calcium enters the cells during the engulfment of apoptotic cells and in turn their expression and function are required for efficient engulfment.

Requirement for the calcium flux machinery is evolutionarily conserved. Recently, we identified stim-1 as one of the $\sim 60$ genes that regulate different aspects of corpse removal in the nematode $C$. elegans; however, how stim-1 might regulate engulfment was not addressed. ${ }^{20}$ The ortholog of STIM-1 in Drosophila and mammals has been shown to be a component of the ER that can communicate with plasma membrane CRAC channels to promote calcium entry into cells. ${ }^{21,22}$ Orai subunits that form the CRAC channel exist basally as a dimer until bound by Stim, resulting in tetramerization and formation of an active channel. ${ }^{19}$ As the studies described above suggested a role for CRAC channels for optimal engulfment, we addressed the phenotype of disrupting stim-1 in the nematode more closely. Knockdown of stim-1 increased the numbers of refractile cell corpses in the adult hermaphrodite gonad (Figure 4a). However, these corpses could represent a defect in apoptosis, phagocytosis, or corpse degradation. ${ }^{20}$ To more strictly define the role of stim-1, we looked at 'actin halo' formation, which represents the enrichment of actin in the phagocyte at sites of active apoptotic cell internalization. ${ }^{20,23}$ Consistent with phagocytic defects, we found a severe decrease in the number of actin halos in stim-1 RNAitreated worms (Figure $4 \mathrm{~b}$ ). This result was quite comparable to the phenotype seen with the knockdown of ced-1, which codes for a potential phagocytic receptor. ${ }^{20}$ Control siRNAtreated worms had very few unengulfed corpses (Figure $4 \mathrm{a}$
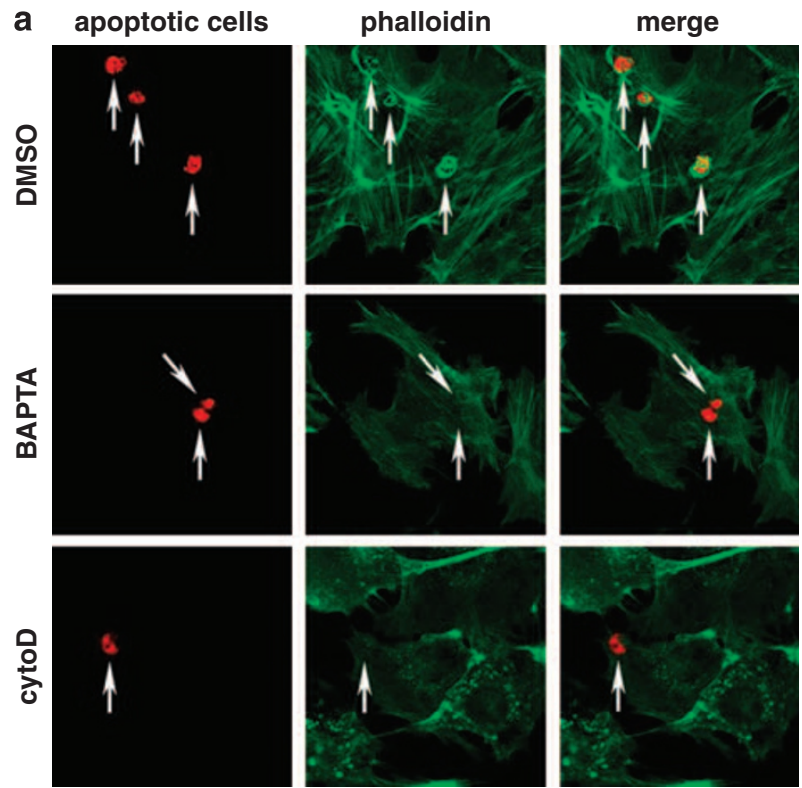

b

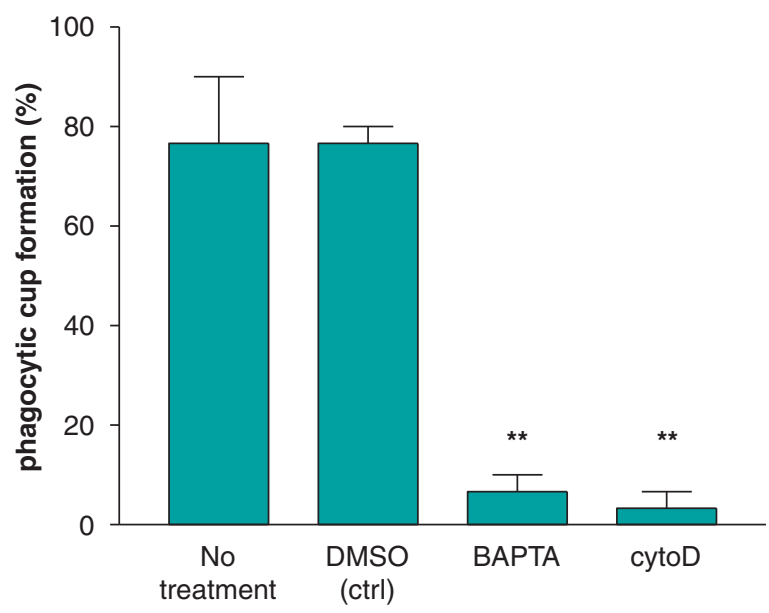

Figure 2 Calcium is important for actin polymerization and phagocytic cup formation. (a) Representative photographs of actin polymerization during apoptotic cell engulfment. (b) Quantitation of the percentage of apoptotic cells that displayed phagocytic cup formation. Apoptotic cells were labeled with TAMRA and incubated with NIH/ ЗT3 cells treated with nothing, vehicle (DMSO), BAPTA, or the actin polymerization inhibitor cytochalasin D. Actin was stained with Alexa 488-phalloidin. Significance is indicated as follows: $P>0.05$, ns; ${ }^{\star} P=0.01-0.05 ;{ }^{\star \star} P=0.001-0.01 ;{ }^{* \star *} P<0.001(n=10)$

Figure 3 Both internal calcium stores and cell membrane calcium channels like the CRAC channels are important for the engulfment of apoptotic cells. (a) LR73 cells were incubated with labeled apoptotic thymocytes in the presence or absence of the indicated drugs and scored in the engulfment assay. Drugs shown were added at the same time as apoptotic thymocytes to minimize gross effects on the phagocytes. This is a representative of three independent experiments. (b) ORAI 1,2, and 3 transcript levels in $\mathrm{NIH} 3 \mathrm{~T} 3$ cells transfected with siRNA specific for the genes indicated on the $x$-axis as measured by real-time PCR. (c) NIH3T3 that were transfected with the indicated siRNA were incubated with labeled apoptotic thymocytes and analyzed for engulfment. (b and $\mathbf{c})$ Representative of two independent experiments. Significance is indicated as follows: $P>0.05$, ns; ${ }^{*} P=0.01-0.05 ;{ }^{* *} P=0.001-0.01 ;{ }^{* *} P<0.001$ 

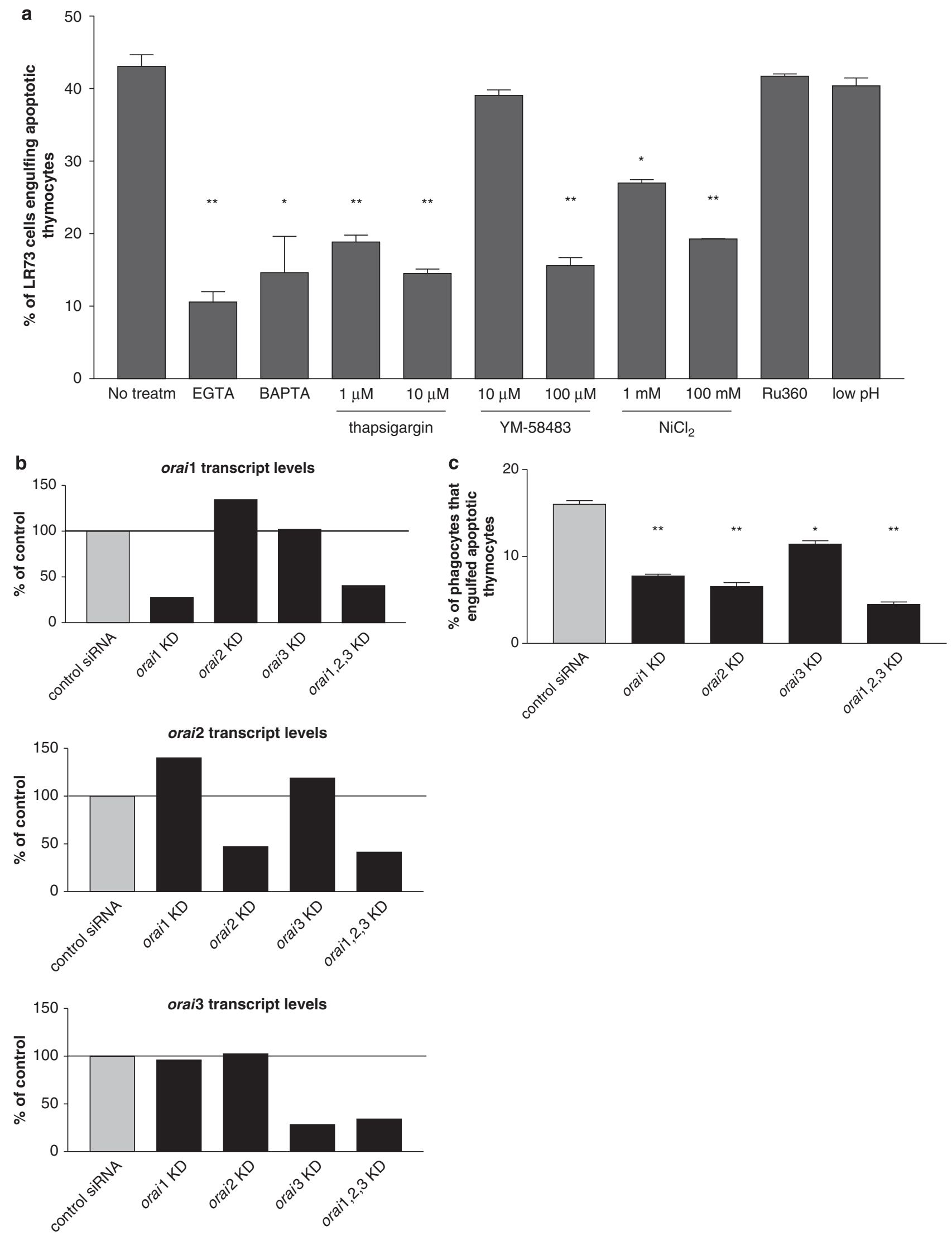


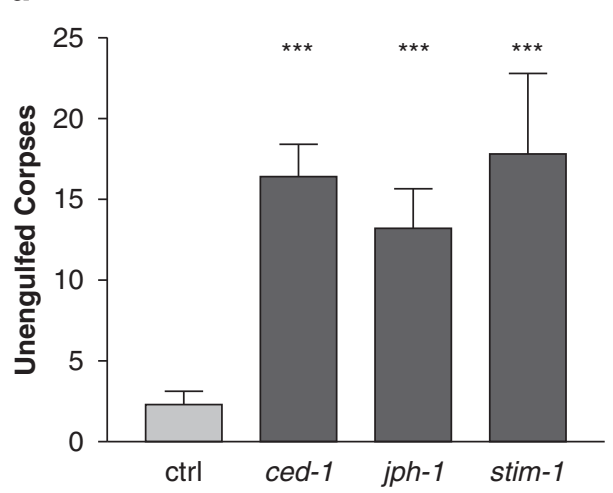

c

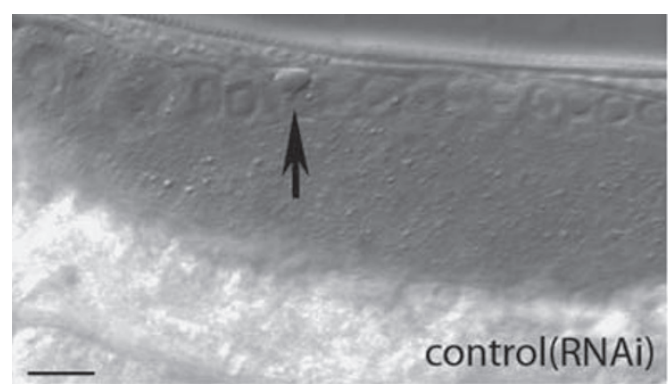

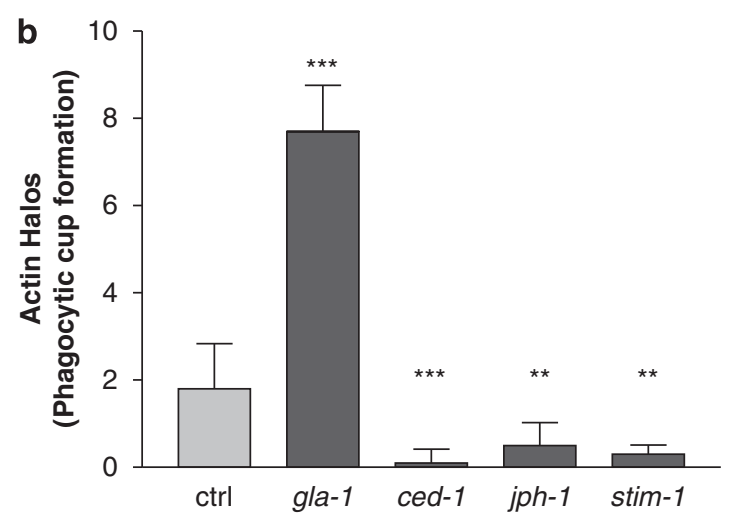

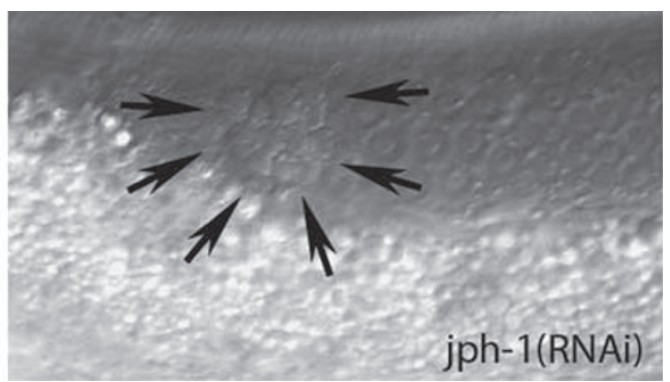

Figure 4 jph-1 and stim-1 are important for corpse clearance in C. elegans. (a) Quantitation of the number of undegraded refractile corpses in the gonad of worms after RNAi knockdown of the indicated genes. Control worms were maintained on bacteria transformed with an RNAi vector containing no insert. (b) Defective corpse internalization in jph-1(RNAi) and stim1(RNAi) worms was determined by scoring the number of actin halos in worms with RNAi knockdown of the indicated genes. Lack of halos in ced-1(RNAi) worms served as the positive control for defects in corpse internalization. Significance is indicated as follows: $P>0.05, n s ;{ }^{*} P=0.01-0.05 ;{ }^{* *} P=0.001-0.01$; ${ }^{* * *} P<0.001$. (c) DIC micrographs showing the $C$. elegans adult hermaphrodite gonad in control (left) or the increased number of corpses in jph-1-deficient animals. Arrows point to refractile cell corpses

and c), whereas gla-1-deficient worms showed increased numbers of halos, consistent with an apoptotic defect. ${ }^{20}$ Taken together, these studies suggest that the importance of CRAC channel activity is evolutionarily conserved.

In an independent screen for regulators of engulfment in Drosophila, a recent study identified a junctophilin-related molecule and further showed a role for dStim in apoptotic cell clearance. ${ }^{8}$ Junctophilins mediate the apposition between the ER and the plasma membrane and are thought to link emptying of intracellular calcium stores to the opening of plasma membrane calcium channels allowing calcium entry into cells. ${ }^{24}$ RNAi-mediated knockdown of the junctophilin homolog jph-1 in $C$. elegans resulted in defective engulfment with an accumulation of a large number of uninternalized refractive corpses (Figure $4 \mathrm{a}$ and $\mathrm{C}$ ). We used RNAi for all of the $C$. elegans experiments, as a Stim-1 mutant is not available, and the Jph-1 mutant worms have severe developmental defects that precluded the analysis of normal engulfment of dying cells in this strain. Collectively, these data suggest a role for the components of the calcium flux machinery in apoptotic cell clearance in the context of a whole organism. These data also suggest that the requirement for calcium during early stages of engulfment is evolutionarily conserved.

Anti-inflammatory cytokine production induced by apoptotic cells requires intracellular calcium flux in phagocytes. Phagocytes engulfing apoptotic cells secrete anti-inflammatory cytokines such as TGF- $\beta .{ }^{25}$ Earlier studies have suggested that secretion of TGF- $\beta$ is separable from the physical engulfment of the corpse, such that 'recognition' of apoptotic cells by the phagocyte induces TGF- $\beta$. For example, the treatment of the phagocytes with the actindisrupting drug, cytochalasin $D$, inhibits internalization of the corpse, but minimally affects TGF- $\beta$ production. ${ }^{5}$ Similarly, 'recognition' of the phosphatidyl serine alone is sufficient to induce cytokine secretion by the phagocyte. ${ }^{26}$ Thus, we asked whether calcium flux in phagocytes, besides its role in phagocytic cup formation, would also affect the generation of TGF- $\beta .{ }^{5}$ We incubated murine J774 macrophages with apoptotic thymocytes in the medium with or without calcium and measured the amount of TGF- $\beta$ secretion. The addition of apoptotic cells to phagocytes stimulated high levels of TGF- $\beta$ secretion $(>100 \mathrm{pg} / \mathrm{ml}$ ) after $18 \mathrm{~h}$ of initial encounter with apoptotic cells; however, in the absence of extracellular $\mathrm{Ca}^{2+}$ in the medium, the production of TGF- $\beta$ was severely reduced (Figure $5 \mathrm{a}$ ). It is noteworthy that this defect is likely not because of defects in the recognition of targets by macrophages, as binding between apoptotic cells and phagocytes seems to occur comparably in a medium with or without calcium (Supplementary Figure 2).

Furthermore, we tested the effect of inhibitors of $\mathrm{Ca}^{2+}$ release on TGF- $\beta$ production. Both extracellular and intracellular calcium stores seem important for TGF- $\beta$ secretion by 

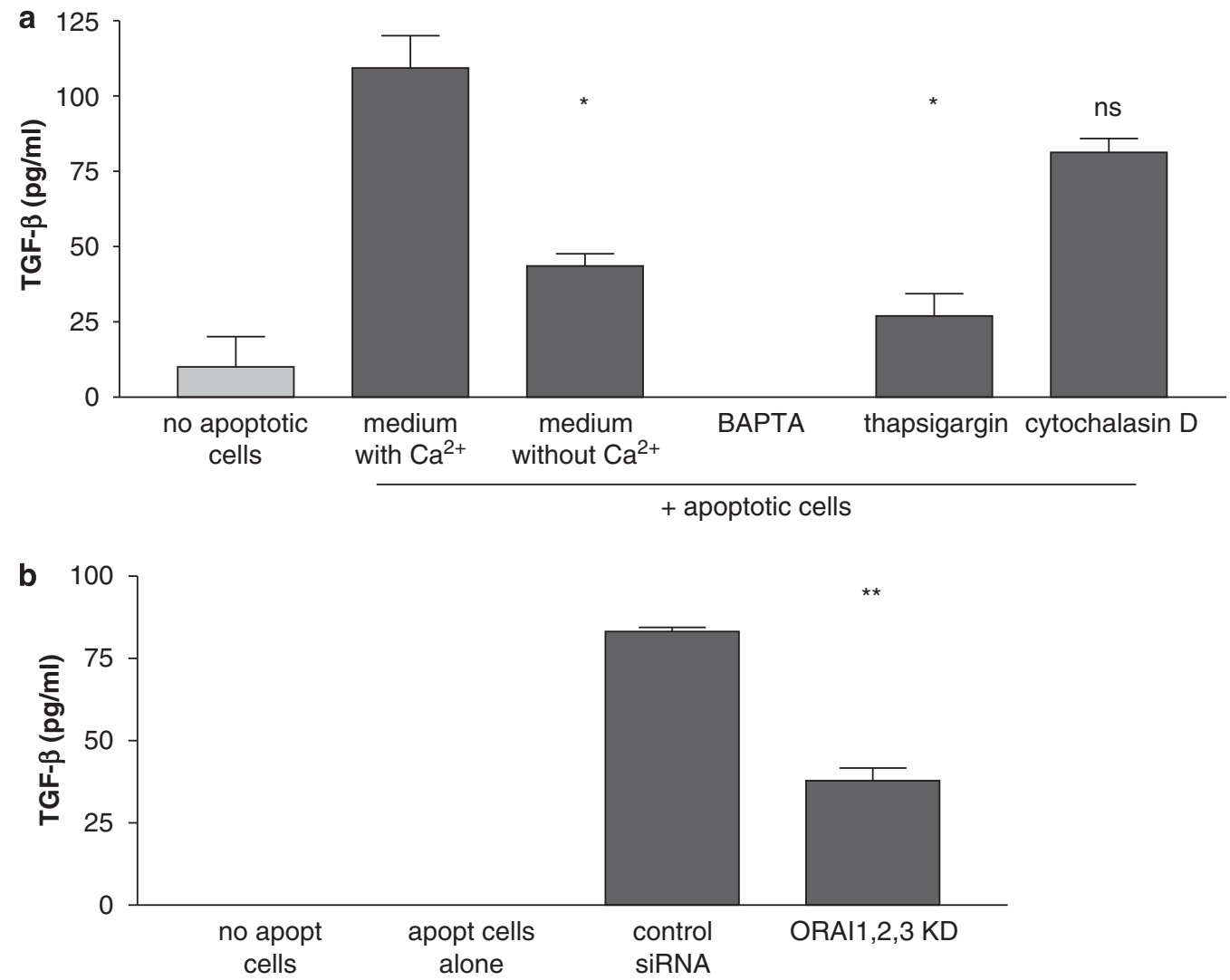

Figure 5 Both intracellular and extracellular $\mathrm{Ca}^{2+}$ is crucial for TGF- $\beta$ secretion by the phagocytes encountering apoptotic cells. (a) J774 phagocytes cells were incubated with apoptotic thymocytes in the presence of absence of calcium in the medium, or in the presence of calcium but with calcium inhibitor drugs. The supernatants were collected and analyzed for TGF- $\beta$ levels by ELISA. Drugs were added at the same time as the apoptotic cells. (b) NIH3T3 cells transfected with control or orai1, orai2, and orai3-specific siRNA were incubated with apoptotic thymocytes and secretion of TGF- $\beta$ was measured in the medium $24 \mathrm{~h}$ later. No apopt cells shows the amount of TGF- $\beta$ secreted by $\mathrm{NIH} 3 \mathrm{~T} 3$ cells without addition of apoptotic thymocytes, whereas apopt cells alone is the amount of TGF- $\beta$ in wells with apoptotic thymocytes without any phagocytes. Each figure is representative of at least three independent experiments. Significance is indicated as follows: $P>0.05$, ns; ${ }^{*} P=0.01-0.05 ;{ }^{* *} P=0.001-0.01 ;{ }^{* * *} P<0.001$

macrophages, as the addition of either BAPTA or thapsigargin resulted in decreased TGF- $\beta$ production (Figure $5 \mathrm{a}$ ). It is noteworthy that under the same conditions, cytochalasin $D$, an actin polymerization inhibitor ${ }^{27}$ that inhibits corpse internalization, ${ }^{28}$ did not have an effect on TGF- $\beta$ production $(P=0.14)$, confirming that engulfment/internalization of the corpse per se is not required for macrophage TGF- $\beta$ production. Intriguingly, the knockdown of all three ORAI homologs in NIH3T3 cells (Figure $3 b$ ) severely reduced the levels of TGF- $\beta$ secretion after incubation with apoptotic thymocytes (Figure 5b) (compared with the nonspecific siRNA-treated control condition).

\section{Discussion}

In this report, we identify the existence of an early calcium flux in phagocytes during the recognition of apoptotic cells and show that this calcium flux is necessary for the engulfment of apoptotic targets. On the basis of in vivo studies in C. elegans, in which downregulation of stim-1 or jph-1 through RNAi inhibits the phagocytosis of apoptotic cells upstream of actin reorganization, coupled with the mammalian studies on the phagocytic cup formation, we propose that the rise in intracellular calcium during apoptotic cell recognition plays a key role in steps leading to the remodeling of the actin cytoskeleton and apoptotic cell internalization. These data also suggest that one or more of the engulfment receptors engaged during apoptotic cell recognition may lead to the activation of intracellular molecules that promote early and sustained calcium release. ${ }^{29-32}$ We also identify some of the important players involved in calcium flux downstream of apoptotic cell recognition, which is likely to have implications for corpse ingestion, as well as potential downstream postengulfment consequences.

Our data also suggest that calcium is required for the postengulfment responses of phagocytes, such as the antiinflammatory response that normally accompanies the engulfment of apoptotic cells. The results presented here suggest that an impaired calcium flux in the phagocytes could predispose an individual to potential autoimmune responses from two sides: reduced clearance of apoptotic cells has been linked to autoimmune phenotypes and a failure of the anti-inflammatory response would further contribute to the inflammation and/or possible autoimmunity. Furthermore, the finding that downstream signaling components for engulfment and the anti-inflammatory response may have both overlapping and distinct players should help further study of divergent downstream signaling. 
Recently, genetic studies in Drosophila proposed roles in the engulfment of Drosophila homologs of mammalian STIM, Orai, and the ryanodine receptor, all genes earlier shown to have a role in calcium flux. ${ }^{8}$ Furthermore, they proposed roles for Draper, a CED-1 homolog, and the adaptor molecule Ced-6, the ortholog of worm CED-6, and mouse GULP in the calcium flux. Using STIM1- or STIM2-deficient MEFs, ${ }^{33}$ we were unable to show defects in the uptake of apoptotic cells (data not shown), potentially because of the compensatory function of one STIM homolog when the other was missing. Furthermore, ryanodine, a specific ryanodine receptor inhibitor, did not inhibit the engulfment in our model (data not shown). Using LRP1 ${ }^{-1-}$ $\mathrm{CHO}$ or GULP knockdown fibroblasts, we also found no essential role for these proteins in calcium flux after the recognition of apoptotic cells (data not shown). Thus, these studies suggest that although a role for calcium flux in corpse removal is evolutionarily conserved, the signal transduction activating this process may be different in mammals and flies.

In our studies, thapsigargin severely inhibited engulfment but not calcium flux. Thapsigargin has been shown earlier to specifically inhibit the SR/ER calcium ATPase,${ }^{34-37}$ supporting our statement that the block in calcium influx from the ER and not some unrelated effect affects engulfment. The 'small' contribution of the ER stores to cytoplasmic calcium levels (as measured by fluorimetry) is also consistent with earlier published literature. ${ }^{33}$ The addition of thapsigargin releases intracellular calcium stores into the cytoplasm, but the net change in calcium levels is barely detectable even in T cells, ${ }^{33}$ which are known to have large calcium fluxes. However, on a cellular level, this change is significant in that it induces a rapid cellular response/downstream signal. Hence, it is not surprising that we see a significant effect on engulfment when treating cells with thapsigargin, yet there is little to no effect on total calcium flux.

\section{Materials and Methods \\ General. Immortalized cell lines and primary cells were maintained as described earlier. ${ }^{38}$ All calcium inhibitory drugs were obtained from Calbiochem. Drugs were used at the following concentrations: BAPTA $10 \mu \mathrm{M}$, EGTA $10 \mathrm{mM}$, thapsigargin 1 or $10 \mu \mathrm{M}$, YM-58483 (BTP-2) 10 or $100 \mu \mathrm{M}, \mathrm{NiCl}_{2} 1-100 \mathrm{mM}$, Ru360 $10 \mu \mathrm{M}$, Cytochalasin D $0.1 \mu \mathrm{M}$ (Supplementary Table 1). All protocols were consistent with accepted National Institutes of Health guidelines for the care and use of laboratory animals and approved by the University of Virginia Institutional Animal Care and Use Committee. Statistical significance was assessed using the $t$-test in Graphpad Prism. Significance is indicated in figures in the following way: $P>0.05$, ns; $0.01-0.05,{ }^{*} ; 0.001-0.01,{ }^{* *} ;<0.001,{ }^{* * *}$.}

In vitro phagocytosis assay. Cell lines (LR73 Chinese hamster ovary cells and $\mathrm{NIH} 3 \mathrm{~T} 3$ cells) were plated overnight in 24-well plates. The targets were apoptotic thymocytes, apoptotic Jurkat, or SCl cells labeled with TAMRA-SE (Invitrogen) as described earlier ${ }^{29}$ and added to the plated cells for $2 \mathrm{~h}$. Apoptotic Jurkat and $\mathrm{SCl}$ cells were generated by incubating them in $5 \mu \mathrm{M}$ Camptothecin (Sigma) overnight, followed by washing. Apoptosis in thymocytes was induced by incubation in $5 \mu \mathrm{M}$ dexamethasone for $4 \mathrm{~h}$. The flow cytometry-based engulfment assay was performed as described earlier. ${ }^{39}$

Calcium measurement assay. LR73 cells were plated on 96-well white opaque plates overnight. The cells were labeled with $1 \mu \mathrm{M}$ Fluo-4 (Invitrogen) in Fluorimetry Media (10 mM Hepes, $150 \mathrm{mM} \mathrm{NaCl}, 5 \mathrm{mM} \mathrm{KCl}, 1 \mathrm{mM} \mathrm{MgCl}, 1 \mathrm{mM}$ $\mathrm{CaCl}_{2}, 0.1 \%$ glucose and $1 \% \mathrm{FBS}$ ) for $1 \mathrm{~h}$. Apoptotic Jurkats were prepared as explained above. Drugs targeting the calcium machinery in the phagocytes were added to the plate $5 \mathrm{~min}$ before the apoptotic cells. A small volume $(10 \mu \mathrm{l})$ of apoptotic cells was added to prevent any changes in the temperature of the medium of LR73 cells. Changes in intracellular calcium levels were measured using Synergy HT microplate reader (Bio-Tek).

C. elegans studies. Feeding RNAi was performed as described ${ }^{40}$ with the following modifications: NGM-agarose plates containing 2 mM IPTG (Eppendorf) and $100 \mu \mathrm{g} / \mathrm{ml}$ carbenocillin (Teknova) were inoculated with $\sim 150 \mu \mathrm{l}$ of appropriate bacterial cultures (transformed with constructs for the generation of double-stranded RNA under the control of the T7 promoter) and were incubated for $8-12 \mathrm{~h}$ before the

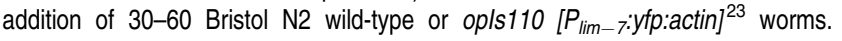
Hypochlorite synchronized $L 1$ stage worms were placed on each RNAi plate and left for $\sim 72 \mathrm{~h}$ at $20^{\circ} \mathrm{C}$. Worms fed with $\mathrm{HT} 115(\mathrm{DH} 3)$ bacteria transformed with the original L4440 RNAi vector containing no insert were used as a control strain. The jph-1 RNAi construct was obtained from the Ahringer RNAi library and was confirmed by sequencing; an $\sim 600 \mathrm{bp}$ stim-1 fragment was amplified from N2 genomic DNA (gcatggtacccaattgaaattccttcaaaaaccatacc and gcatgcggccgcggcaaat gcgctctatcggca) adding Kpnl and Notl restriction sites and then subcloned into

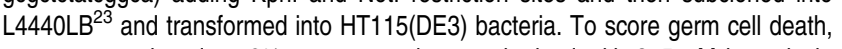
worms were placed on $2 \%$ agarose pads, anesthetized with 3-5 mM levamisole (Sigma), and mounted under a coverslip for observation using a Zeiss Axiovert 200 microscope equipped with DIC (Nomarski) optics and standard epifluorescence filter sets appropriate for the visualization of YFP. The number of germ cell corpses and/or actin fluorescence 'halos' was then scored by DIC or fluorescence microscopy as described earlier. ${ }^{23}$ Bacteria transformed with the original L444O(RNAi) vector containing no insert were used as a reference strain.

Immunofluorescence microscopy of mammalian cells. $\mathrm{NIH} / 3 \mathrm{~T} 3$ fibroblasts were plated on LabTekll culture chambers (Fisher) in DMEM 10\% FBS overnight, then incubated with $1 \times 10^{6}$ apoptotic SCl cells for $30 \mathrm{~min}$. Images were acquired using a Zeiss 510-UV laser scanning confocal microscope with 488 and 543 lasers (Zeiss AG). Cells were then fixed with $3 \%$ paraformaldehyde (Sigma) in PBS for 30 min, permeabilized with $0.1 \%$ Triton X-100 (Sigma) and blocked with $5 \%$ skim milk that had been clarified by high-speed centrifugation. The cells were subsequently stained with Alexa 488 -phalloidin as described earlier. ${ }^{41}$

Knockdown. NIH3T3 cells were transfected with $\sim 1.2 \mu \mathrm{g}$ siRNA (Dharmacon cat\#: orai1 M-056431-00, orai2 M-057985-00, orai3 M-054417-00, control siRNA M-001206-13-05) using an Amaxa nucleofector (Amaxa Biosystems) according to the manufacturer's specifications (kit R, program U-30) (as described earlier ${ }^{20}$ ). Knockdown was confirmed using real-time PCR. First, RNA was extracted using the RNeasy mini kit (Qiagen) and reverse transcribed using Superscript III first strand kit (Invitrogen). Real-time PCR was carried out using the StepOne Plus RT-PCR System using Taqman probes (both from Applied Biosystems). GAPDH and 18S served as endogenous controls. Analysis was made using StepOne v2.0 software (Applied Biosystems).

TGF- $\beta$ secretion assay. J774 macrophages or siRNA-transfected NIH3T3 were plated in a 12 -well plate at $37^{\circ} \mathrm{C}$. After 1 or 2 days , $2 \times 10^{6}$ apoptotic thymocytes (generated as described above) were co-incubated for $18 \mathrm{~h}$ with the $\mathrm{J} 774$ cells in a culture medium (X-VIVO 10) with or without $\mathrm{Ca}^{2+}$ or the indicated $\mathrm{Ca}^{2+}$-specific inhibitors. The culture supernatants were then collected, centrifuged, and analyzed for total TGF- $\beta$ using the TGF- $\beta$ ELISA/assay kit (R\&D Systems) following the manufacturer's instructions.

Acknowledgements. We thank the members of the Ravichandran and Franc labs and Dr. D Haverstick for helpful discussions. We gratefully acknowledge the help of Marcin Iwanicki with the calcium experiments. This work was supported by grants from the National Institutes of Health (NIGMS) and American Asthma Foundation to KSR, and by post-doctoral fellowships from the Natural Sciences and Engineering Research Council of Canada (NSERC) to MAG and the Arthritis Foundation to JMK, as well as Immunology Training Grants from the NIAID (National Institutes of Health, USA) to IJJ and JMK.

\section{Conflict of interest}

The authors declare no conflict of interest.

1. Erwig LP, Henson PM. Immunological consequences of apoptotic cell phagocytosis. Am J Pathol 2007; 171: 2-8. 
2. Monks J, Smith-Steinhart C, Kruk ER, Fadok VA, Henson PM. Epithelial cells remove apoptotic epithelial cells during post-lactation involution of the mouse mammary gland. Biol Reprod 2008; 78: 586-594.

3. Savill J, Dransfield I, Gregory C, Haslett C. A blast from the past: clearance of apoptotic cells regulates immune responses. Nat Rev Immunol 2002; 2: 965-975.

4. Erwig LP, Henson PM. Clearance of apoptotic cells by phagocytes. Cell Death Differ 2008; 15: $243-250$

5. Cvetanovic M, Ucker DS. Innate immune discrimination of apoptotic cells: repression of proinflammatory macrophage transcription is coupled directly to specific recognition J Immunol 2004; 172: 880-889.

6. Lewis RS. Calcium signaling mechanisms in T lymphocytes. Annu Rev Immunol 2001; 19 497-521.

7. Watanabe N, Suzuki J, Kobayashi Y. Role of calcium in tumor necrosis factor-alpha production by activated macrophages. J Biochem 1996; 120: 1190-1195.

8. Cuttell L, Vaughan A, Silva E, Escaron CJ, Lavine M, Van Goethem E et al. Undertaker, a Drosophila Junctophilin, links Draper-mediated phagocytosis and calcium homeostasis Cell 2008; 135: 524-534.

9. Oh-Hora M, Rao A. Calcium signaling in lymphocytes. Curr Opin Immunol 2008; 20: 250-258.

10. Feske S. Calcium signalling in lymphocyte activation and disease. Nat Rev Immunol 2007; 7: 690-702.

11. Hynes RO. Integrins: bidirectional, allosteric signaling machines. Cell 2002; 110 673-687.

12. Dransfield I, Cabanas $C$, Craig A, Hogg N. Divalent cation regulation of the function of the leukocyte integrin LFA-1. J Cell Biol 1992; 116: 219-226.

13. Tosello-Trampont AC, Brugnera E, Ravichandran KS. Evidence for a conserved role for CRKII and Rac in engulfment of apoptotic cells. J Biol Chem 2001; 276: 13797-13802.

14. Hajnoczky G, Csordas G, Das S, Garcia-Perez C, Saotome M, Sinha RS et at. Mitochondrial calcium signalling and cell death: approaches for assessing the role of mitochondrial $\mathrm{Ca}^{2+}$ uptake in apoptosis. Cell Calcium 2006; 40: 553-560.

15. Kang HW, Park JY, Jeong SW, Kim JA, Moon HJ, Perez-Reyes E et al. A molecular determinant of nickel inhibition in Cav3.2 T-type calcium channels. J Biol Chem 2006; 281 : 4823-4830.

16. Kimura J, Miyamae S, Noma A. Identification of sodium-calcium exchange current in single ventricular cells of guinea-pig. J Physiol 1987; 384: 199-222.

17. Yoshino T, Ishikawa J, Ohga K, Morokata T, Takezawa R, Morio H et al. YM-58483, selective CRAC channel inhibitor, prevents antigen-induced airway eosinophilia and late phase asthmatic responses via Th2 cytokine inhibition in animal models. Eur J Pharmacol 2007; 560: 225-233

18. Zitt C, Strauss B, Schwarz EC, Spaeth N, Rast G, Hatzelmann A et al. Potent inhibition of $\mathrm{Ca}^{2+}$ release-activated $\mathrm{Ca}^{2+}$ channels and T-lymphocyte activation by the pyrazole derivative BTP2. J Biol Chem 2004; 279: 12427-12437.

19. Penna A, Demuro A, Yeromin AV, Zhang SL, Safrina O, Parker I et al. The CRAC channel consists of a tetramer formed by Stim-induced dimerization of Orai dimers. Nature 2008; 456: 116-120.

20. Kinchen JM, Doukoumetzidis K, Almendinger J, Stergiou L, Tosello-Trampont A, Sifri CD et al. A pathway for phagosome maturation during engulfment of apoptotic cells. Nat Cell Biol 2008; 10: 556-566.

21. Roos J, DiGregorio PJ, Yeromin AV, Ohlsen K, Lioudyno M, Zhang S et al. STIM1, an essential and conserved component of store-operated $\mathrm{Ca}^{2+}$ channel function. J Cell Bio 2005; 169: 435-445.
22. Liou J, Kim ML, Heo WD, Jones JT, Myers JW, Ferrell Jr JE et al. STIM is a $\mathrm{Ca}^{2+}$ sensor essential for $\mathrm{Ca}^{2+}$-store-depletion-triggered $\mathrm{Ca}^{2+}$ influx. Curr Biol 2005; 15: 1235-1241.

23. Kinchen JM, Cabello J, Klingele D, Wong K, Feichtinger R, Schnabel H et al. Two pathways converge at CED-10 to mediate actin rearrangement and corpse removal in $\mathrm{C}$. elegans. Nature 2005; 434: 93-99.

24. Takeshima H, Komazaki S, Nishi M, lino M, Kangawa K. Junctophilins: a novel family of junctional membrane complex proteins. Mol Cell 2000; 6: 11-22.

25. Fadok VA, Bratton DL, Konowal A, Freed PW, Westcott JY, Henson PM. Macrophages that have ingested apoptotic cells in vitro inhibit proinflammatory cytokine production through autocrine/paracrine mechanisms involving TGF-beta, PGE2, and PAF. J Clin Invest 1998; 101: 890-898.

26. Kim S, Elkon KB, Ma X. Transcriptional suppression of interleukin-12 gene expression following phagocytosis of apoptotic cells. Immunity 2004; 21: 643-653.

27. Casella JF, Flanagan MD, Lin S. Cytochalasin D inhibits actin polymerization and induces depolymerization of actin filaments formed during platelet shape change. Nature 1981; 293: 302-305.

28. Park D, Hochreiter-Hufford A, Ravichandran KS. The phosphatidylserine receptor TIM-4 does not mediate direct signaling. Curr Biol 2009; 19: 346-351.

29. Park D, Tosello-Trampont AC, Elliott MR, Lu M, Haney LB, Ma Z et al. BAl1 is an engulfment receptor for apoptotic cells upstream of the ELMO/Dock180/Rac module. Nature 2007; 450: 430-434

30. Miyanishi M, Tada K, Koike M, Uchiyama Y, Kitamura T, Nagata S. Identification of Tim4 as a phosphatidylserine receptor. Nature 2007; 450: 435-439.

31. Park SY, Jung MY, Kim HJ, Lee SJ, Kim SY, Lee BH et al. Rapid cell corpse clearance by stabilin-2, a membrane phosphatidylserine receptor. Cell Death Differ 2008; 15: 192-201.

32. Gardai SJ, Bratton DL, Ogden CA, Henson PM. Recognition ligands on apoptotic cells: a perspective. J Leukoc Biol 2006; 79: 896-903.

33. Oh-Hora M, Yamashita M, Hogan PG, Sharma S, Lamperti E, Chung W et al. Dual functions for the endoplasmic reticulum calcium sensors STIM1 and STIM2 in T cell activation and tolerance. Nat Immunol 2008; 9: 432-443.

34. Rogers TB, Inesi G, Wade R, Lederer WJ. Use of thapsigargin to study $\mathrm{Ca}^{2+}$ homeostasis in cardiac cells. Biosci Rep 1995; 15: 341-349.

35. Kijima $\mathrm{Y}$, Ogunbunmi $\mathrm{E}$, Fleischer $\mathrm{S}$. Drug action of thapsigargin on the $\mathrm{Ca}^{2+}$ pump protein of sarcoplasmic reticulum. J Biol Chem 1991; 266: 22912-22918.

36. Lytton J, Westlin M, Hanley MR. Thapsigargin inhibits the sarcoplasmic or endoplasmic reticulum Ca-ATPase family of calcium pumps. J Biol Chem 1991; 266: 17067-17071.

37. Toyoshima $\mathrm{C}$, Nomura $\mathrm{H}$. Structural changes in the calcium pump accompanying the dissociation of calcium. Nature 2002; 418: 605-611.

38. Brugnera E, Haney L, Grimsley C, Lu M, Walk SF, Tosello-Trampont AC et al. Unconventional Rac-GEF activity is mediated through the Dock180-ELMO complex. Nat Cell Biol 2002; 4: 574-582.

39. Tosello-Trampont AC, Kinchen JM, Brugnera E, Haney LB, Hengartner MO, Ravichandran KS. Identification of two signaling submodules within the Crkll/ELMO/ Dock180 pathway regulating engulfment of apoptotic cells. Cell Death Differ 2007; 14: 963-972

40. Kamath RS, Fraser AG, Dong Y, Poulin G, Durbin R, Gotta M et al. Systematic functional analysis of the Caenorhabditis elegans genome using RNAi. Nature 2003; 421: 231-237.

41. Grimsley CM, Lu M, Haney LB, Kinchen JM, Ravichandran KS. Characterization of a nove interaction between ELMO1 and ERM proteins. J Biol Chem 2006; 281: 5928-5937.

\section{Supplementary Information accompanies the paper on Cell Death and Differentiation website (http://www.nature.com/cdd)}

